\title{
The issue of dynamic hyperinflation in acute respiratory distress syndrome patients
}

\author{
A. Vieillard-Baron, F. Jardin
}

The issue of dynamic hyperinflation in acute respiratory distress syndrome patients. A. Vieillard-Baron, F. Jardin. (C) ERS Journals Ltd 2003.

ABSTRACT: Dynamic hyperinflation is produced by a diffuse expiratory flow limitation impairing exhalation under mechanical ventilation. It constitutes a serious clinical problem in patients exhibiting bronchial asthma or chronic obstructive pulmonary disease, when mechanical ventilation is required. But this phenomenon may also complicate respiratory support in acute respiratory distress syndrome (ARDS) patients.

The presence of diffuse airflow limitation in ARDS patients has sometimes been noticed in the past, but its consequences have only recently been emphasised in a dynamic study, using bedside recording of flow/volume loops during respiratory support.

More recently, by recording systematically a prolonged expiration, the current authors have observed a localised airflow limitation in a majority of ARDS patients, constituting another potential factor of dynamic hyperinflation under respiratory support. In the same report, the current authors' have emphasised the impact of this limitation on the shape of the pressure/volume loop.

At a time when increasing respiratory support is proposed to improve carbon dioxide clearance in acute respiratory distress syndrome submitted to protective ventilation, dynamic hyperinflation may become a major clinical problem in this setting. Eur Respir J 2003; 22: Suppl. 42, 43s-47s.
Medical Intensive Care Unit, University Hospital Ambroise Paré, Assistance Publique Hôpitaux de Paris, Boulogne, France.

Correspondence: F. Jardin

Hôpital Ambroise Paré

9 avenue Charles de Gaulle

92104, Boulogne Cedex

France

Fax: 33149095892

E-mail: francois.jardin@apr.ap-hop-paris.fr

Keywords: Acute respiratory distress syndrome dynamic hyperinflation

intrinsic positive end-expiratory pressure

slow compartment
A temporary incomplete exhalation of the selected tidal volume during mechanical ventilation produces dynamic hyperinflation. Resulting gas trapping initially enlarges lung volume, which rapidly stabilises at a higher value. With the generated increase in elastic recoil, the entire tidal volume can thus be exhaled again.

The presence of dynamic hyperinflation can be detected in a patient by occluding the expiratory port at end-exhalation and recording airway pressure. This manoeuvre permits the observation that alveolar pressure has not returned to zero. The remaining positive end-expiratory alveolar pressure is called "auto-PEEP", or "intrinsic PEEP" (PEEPi).

Dynamic hyperinflation is produced by a diffuse expiratory flow limitation, which may be absolute, or relative to the expiratory time, or by a localised airflow limitation.

\section{Diffuse expiratory flow limitation}

The initial description of diffuse expiratory flow limitation can be attributed to PEPE and MARINI [1]. In their study, these authors observed that, in two chronic obstructive pulmonary disease (COPD) patients ventilated mechanically, alveolar pressure cannot return to zero at the end of tidal exhalation, resulting in PEEPi. From a physiological point of view, PEEPi is generated when three to four time constants cannot elapse between ventilatory cycles [1] (fig. 1). Because the time constant is the product of total compliance and airway resistance, occurrence of PEEPi is expected when compliance is increased, as in emphysema, or when airway resistance is increased, as in bronchial obstruction, both being associated in COPD and producing a marked increase in the time constant. Conversely, in acute respiratory distress syndrome (ARDS) patients, the marked reduction in compliance may protect against PEEPi. However, expiratory flow limitation has been recently described in ARDS patients, and, if not totally compensated for by decreased compliance, it may constitute a potential cause of PEEPi [2].

In their initial report, PEPE and MARINI [1] also observed PEEPi in a patient free of bronchial disease. This finding was interpreted as the result of an excessive respiratory rate under mechanical ventilation, leading to an expiratory time insufficient for a complete exhalation, despite a likely normal time constant. In ARDS patients, several studies have illustrated the frequency of diffuse dynamic hyperinflation produced by an excessive respiratory rate $[3,4,5]$. In the same manner, use of an inverse inspiratory/expiratory ratio also produces dynamic hyperinflation [6].

The pattern of PEEPi produced by diffuse expiratory flow limitation in COPD patients, or in ARDS patients ventilated with an excessive rate, is characteristic, with a sharp increase and a rapid stabilisation of airway pressure at the end of exhalation, as soon as the expiratory port is occluded (fig. 2).

\section{Localised expiratory flow limitation}

The present authors have recently described localised expiratory flow limitation in ARDS patients [7]. In these patients, whereas the average time constant is not increased, the presence of a "slow compartment", with a specifically elevated time constant, produces a localised gas trapping. In other words, it is the uneven distribution of the time constant responsible for a "slow compartment" associated with a "fast 


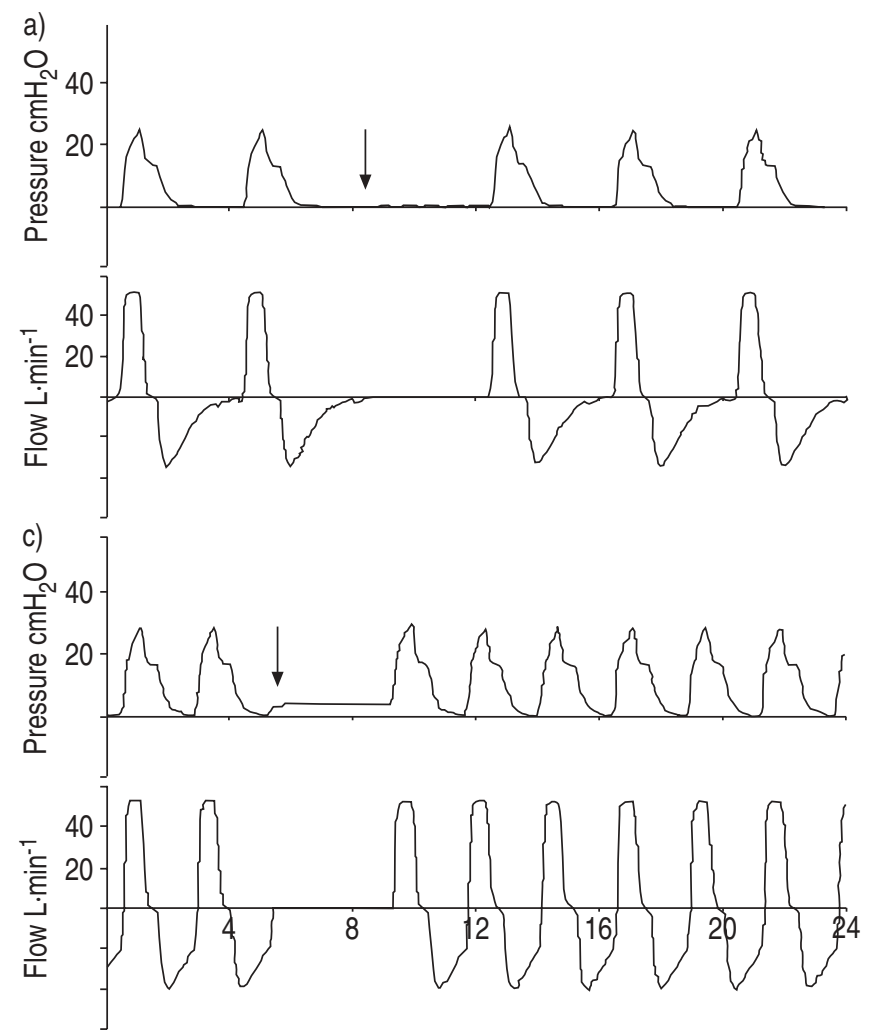

Time s b)

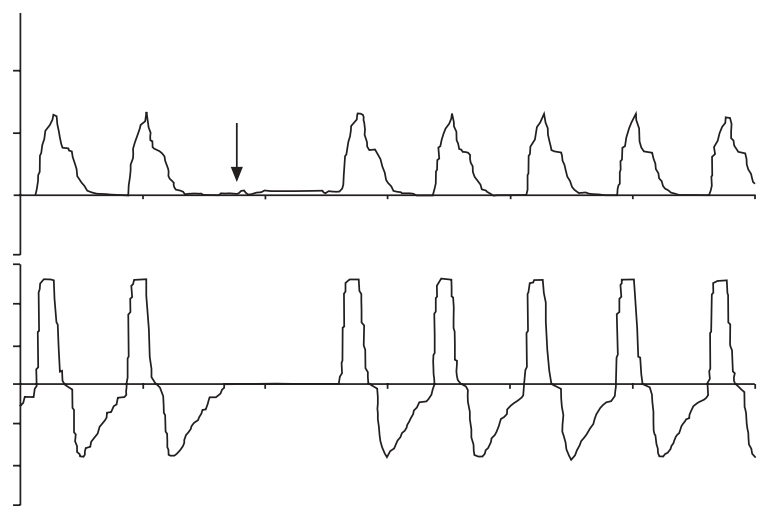

d)

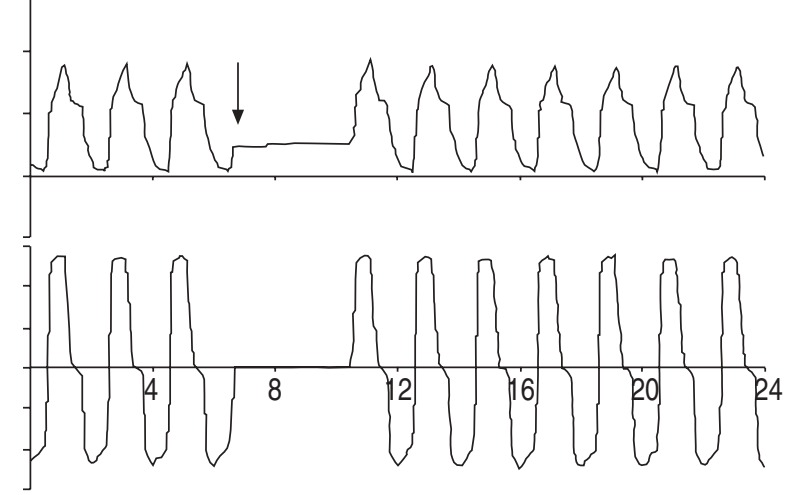

Time s

Fig. 1. - In this patient with an acute lung injury, a progressive increase in respiratory rate under mechanical ventilation caused dynamic hyperinflation when expiration duration was progressively reduced to below three times the time constant (the large vertical arrows denote endexpiratory occlusion). a) The initial ventilator setting includes a constant inspiratory flow $\left(50 \mathrm{~L} \cdot \mathrm{min}^{-1}\right)$, a tidal volume of $550 \mathrm{~mL}$, zero endexpiratory pressure, a respiratory rate of 15 cycles $\cdot \mathrm{min}^{-1}$, an inspiratory/expiratory ratio of $1: 2$, leading to an expiratory duration of $2.6 \mathrm{~s}$. The compliance of the respiratory system was calculated as tidal volume/plateau pressure, the resistance of the respiratory system was calculated as (peak pressure-plateau pressure)/flow, resulting in a time constant (the product of compliance by resistance) of $0.52 \mathrm{~s}$. b) When the respiratory cycle was increased to 20 breaths $\mathrm{min}^{-1}$, expiratory duration was shortened to $2 \mathrm{~s}$, still permitting three time constants to elapse. Nevertheless, a low intrinsic positive end-expiratory pressure (PEEPi) $\left(1 \mathrm{cmH}_{2} \mathrm{O}\right)$ was still observed after a prolonged end-expiratory occlusion, reflecting a localised hyperinflation, from an uneven distribution of the time constant. c) When the respiratory cycle was increased to 25 breaths·min $^{-1}$, expiration duration was shortened to $1.6 \mathrm{~s}$, which just corresponds to three time constants, and diffuse dynamic hyperinflation occurred, resulting in a PEEPi of $5 \mathrm{cmH}_{2} \mathrm{O}$. d) An additional reduction of expiration duration to $1.33 \mathrm{~s}$ by increasing respiratory rate to 30 breaths $\cdot \mathrm{min}^{-1}$ was accompanied by a greater PEEPi $\left(10 \mathrm{cmH}_{2} \mathrm{O}\right)$.

compartment" which produces dynamic hyperinflation localised to the "slow compartment". The amount of PEEPi generated by gas trapping is related to the size of this "slow compartment" (fig. 3) [7]. In this setting, PEEPi is usually low (average $3 \mathrm{cmH}_{2} \mathrm{O}$ in the above mentioned study), because it stabilises after dilution of gas trapped in the "fast compartment" during end-expiratory occlusion, and this is reflected by the prolonged time ( $>3 \mathrm{~s}$ ) required to record a plateau during expiratory occlusion (fig. 2). In the above mentioned study, localised flow limitation was also present during inspiration, and was responsible for the lower inflection point (LIP) of the pressure/volume $(P / V)$ curve [7].

The pattern of PEEPi produced by localised expiratory flow limitation in ARDS patients is thus characteristic, with a very progressive increase and a slow stabilisation of airway pressure at the end of exhalation, when the expiratory port is occluded (fig. 2). This specific pattern was first described by BILEN and COHEN [8], but they gave an inadequate explanation of their original finding.

\section{Mechanical consequence of dynamic hyperinflation}

Occurrence of diffuse dynamic hyperinflation in ARDS increases airway pressure and may counteract a protective respiratory strategy [4]. Thus, to be compatible with airway pressure limitation, dynamic hyperinflation requires an additional reduction in tidal volume to limit plateau pressure [4]. Imposed by an inadequate respiratory rate, this reduction limits minute ventilation, a potential factor for unnecessary hypercapnia [4].

When diffuse dynamic hyperinflation is present in an ARDS patient, calculation of quasi-static compliance ( $\mathrm{Crs}$ ) during mechanical ventilation should be corrected for PEEPi [9]. The lack of correction may in part explain the results of the study of SUTER et al. [10], evidencing a "best" PEEP as a PEEP that significantly improves Crs. However, when dynamic hyperinflation is localised to a "slow compartment", the correction for PEEPi is debatable [7]. This correction allows calculation of an average compliance, including both "fast" and "slow" compartments, whereas calculation without correction is only informative about the compliance of the "fast compartment". Because the "fast compartment" probably represents the most damaged areas, where compliance is reduced, its compliance is the most relevant data in assessing altered lung function and in evaluating the impact of respiratory strategy. Suppression of the "slow compartment" by an adequate PEEP improves Crs [7], and this may also explain another aspect of the results of SUTER et al. [10]. This adequate PEEP is, however, related to but regularly higher 


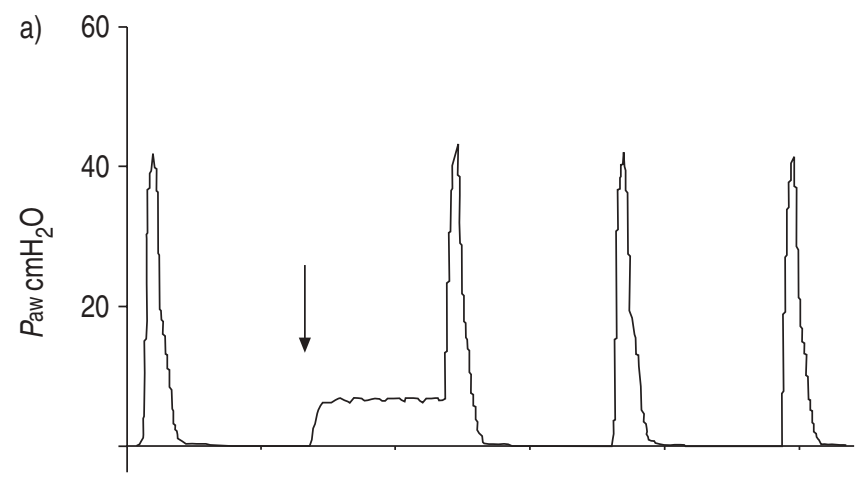

b)

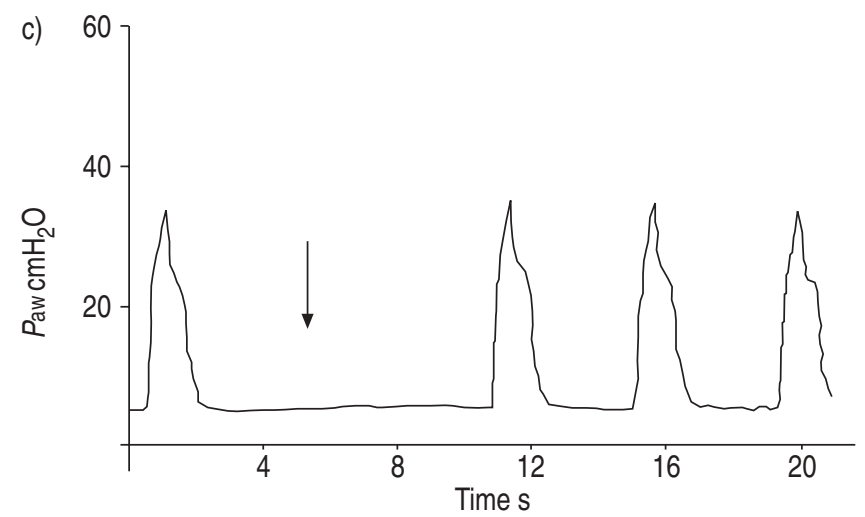

d)
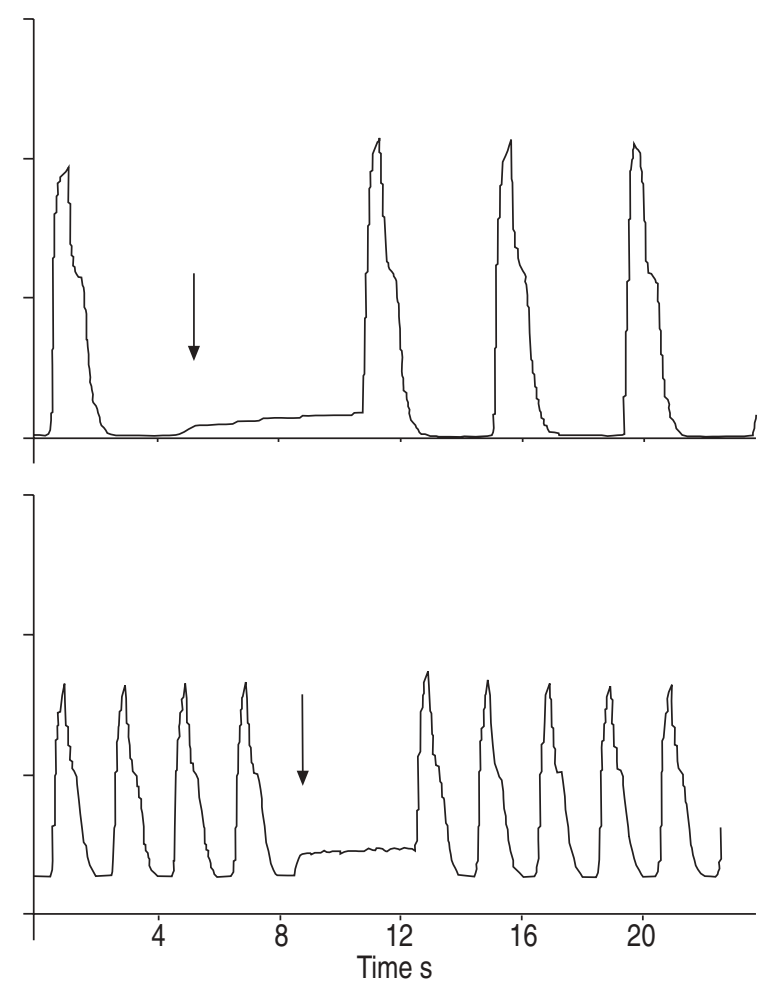

Fig. 2. - Illustrative examples of intrinsic positive end-expiratory pressure (PEEPi) produced by a) and d) diffuse airflow limitation, and b) localised airflow limitation. a) In a chronic obstructive pulmonary disease (COPD) patient, after end-expiratory airway occlusion (vertical arrow), a sharp increase and a quasi-immediate plateau in airway pressure $(P$ aw $)$ was observed, despite a relatively low respiratory rate $(12$ breaths $\left.\cdot \min ^{-1}\right)$. b) In an acute respiratory distress syndrome patient ventilated with a moderate respiratory rate $\left(15\right.$ breaths $\left.\cdot \mathrm{min}^{-1}\right)$ and zero endexpiratory pressure, a very slow rise and a delayed plateau in $P$ aw were observed after end-expiratory airway occlusion (arrow), a typical pattern of PEEPi produced by localised airflow limitation. c) In the same patient, addition of a low external PEEP suppressed PEEPi, as illustrated by the lack of change in $P$ aw after end-expiratory occlusion (arrow). d) In the same patient again, an increase in respiratory rate to 30 breaths·min ${ }^{-1}$ produced diffuse airflow limitation, as illustrated by reappearance of PEEPi after end-expiratory occlusion (vertical arrow). Note that the pattern of PEEPi is the same as in the COPD patient (sharp increase and immediate plateau).

than PEEPi $\left(6 \mathrm{cmH}_{2} \mathrm{O}\right.$ versus $3 \mathrm{cmH}_{2} \mathrm{O}$ on average in the above mentioned study) [7] (fig. 4), and this may be explained by the fact that PEEPi reflects the remaining elastic recoil of the whole respiratory system, when the volume of gas initially trapped in the "slow compartment" has expanded in the "fast compartment". This "pendelluft effect" precludes exact

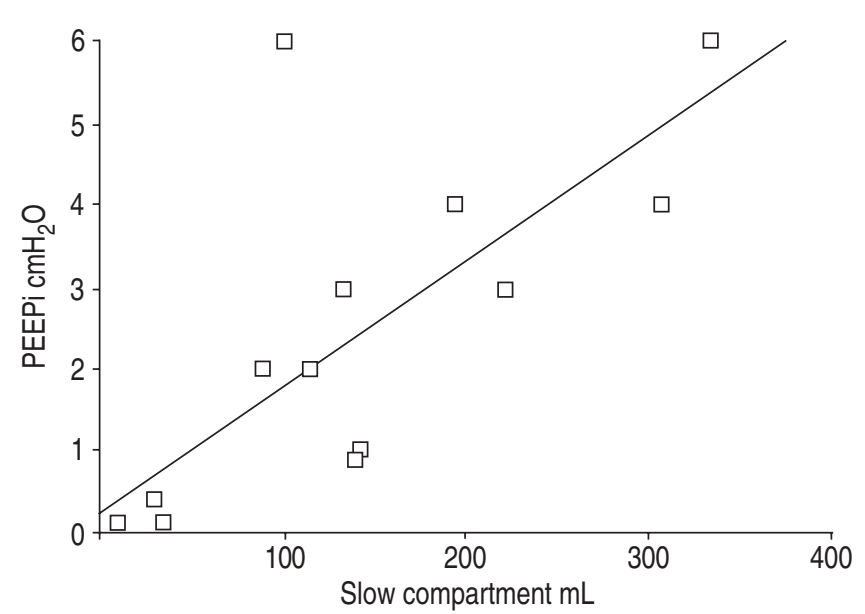

Fig. 3.-Plot of individual values of intrinsic positive end-expiratory pressure (PEEPi), measured at zero end-expiratory pressure, against the size of the "slow compartment", measured during a prolonged exhalation in 16 acute respiratory distress syndrome patients [7]. measurement of the alveolar pressure persisting at endexhalation in the "slow compartment", and any accurate measurement of the specific compliance of this "slow compartment".

As previously stated, the presence of localised dynamic hyperinflation produces a lower inflexion point on the

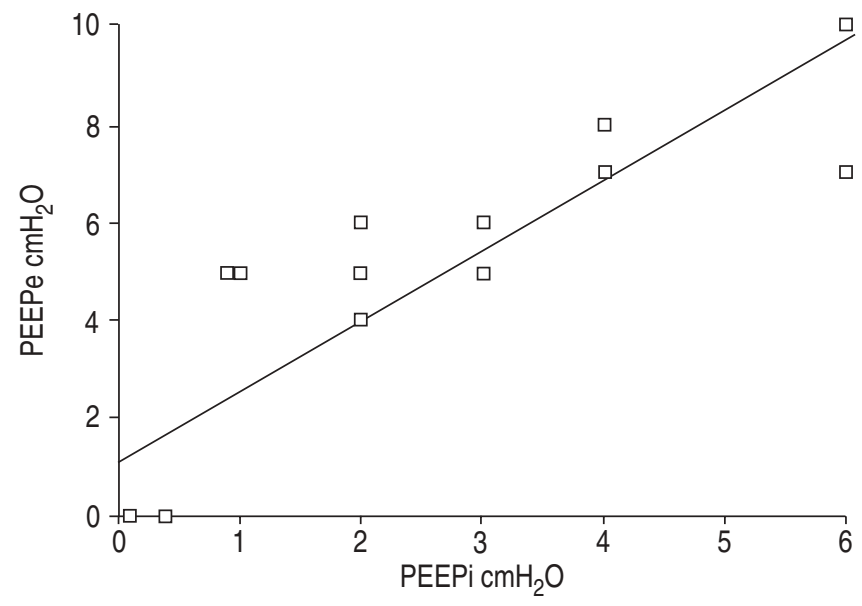

Fig. 4. - Plot of individual values of intrinsic positive end-expiratory pressure (PEEPi) at zero end-expiratory pressure against the external PEEP (PEEPe) required to neutralise PEEPi in 16 acute respiratory distress syndrome patients [7]. (A value of PEEPi $<1 \mathrm{cmH}_{2} \mathrm{O}$ was considered as negligible and was not neutralised by a PEEPe). 
inspiratory $P / V$ curve at zero end-expiratory pressure (ZEEP), with a marked reduction in the starting compliance (i.e. the slope of the first segment of the inspiratory curve) [7], and a large hysteresis between the inspiratory and expiratory limbs of the complete $P / V$ loop [7]. This rightward shift on the inspiratory curve at ZEEP by airflow limitation leads to an overestimation of recruitment, when measured by superimposing the $P / V$ curve obtained at any PEEP on the $P / V$ curve obtained at ZEEP. Application of a PEEP neutralising the "slow compartment" suppresses the LIP and corrects the shift of the P/V curve $[7,10]$. The PEEP required to neutralise the "slow compartment" is regularly lower than the pressure coordinate of the LIP (Pflex) [7]. This constitutes a clinical illustration of a well-known physiological concept [11]: for a given small airway, "opening pressure", that is the pressure required to open the cavity when it is collapsed ( $P$ flex in this example), is always higher than the "closing pressure", that is the pressure required to prevent collapse (PEEP required to neutralise the "slow compartment" in this example) (fig. 5). The current authors' have previously illustrated the application of this mechanical concept in ARDS patients [12].

\section{Haemodynamic consequence of dynamic hyperinflation}

When diffuse, dynamic hyperinflation increases both pleural pressure [1], which impairs right ventricular filling, and pulmonary vascular resistance, which impairs right ventricular outflow [4]. The net result is a drop in cardiac output. This adverse haemodynamic effect has been documented in COPD patients [1], and in ARDS patients ventilated with an inverse inspiratory/expiratory ratio [13], or with an elevated respiratory rate [4]. From a practical point of view, diffuse dynamic hyperinflation during mechanical ventilation should be corrected as far as possible.

Use of an external PEEP, which causes a greater increase in end-expiratory lung volume and cannot totally suppress

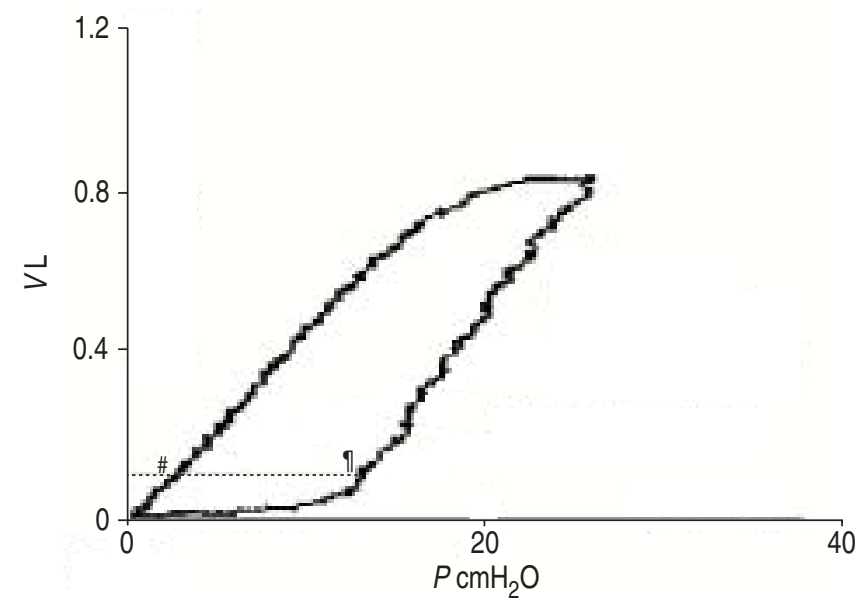

Fig. 5. $-\mathrm{A}$ complete low flow $\left(<10 \mathrm{~L} \cdot \mathrm{min}^{-1}\right)$ pressure/volume $(P / V)$ curve obtained in an acute respiratory distress syndrome patient. This curve shows the marked hysteresis between the inspiratory (right ascending part) and the expiratory (left descending part) limbs produced by the "slow compartment", and illustrates the difference between the pressure required to open the closed distal airways of this "slow compartment" ( $\%$ opening pressure or Pflex) and the pressure sufficient to prevent this closure $(\#$ : closing pressure), thus neutralising the slow compartment. The horizontal dotted line represents an identical inflated (inspiration), or not deflated (expiration) volume above which occluded airways open (inspiration; $"$ ), or close (expiration; ${ }^{\#}$ ).
PEEPi, except by a marked increase in total PEEP (i.e. PEEPi plus external PEEP) [14, 15], is not an adequate way of correcting the haemodynamic consequence of diffuse dynamic hyperinflation. On the contrary, use of an external PEEP in this setting worsens the drop in cardiac output [14, 15]. Only a marked increase in expiratory time may limit diffuse dynamic hyperinflation and its adverse haemodynamic consequences [1].

When localised, dynamic hyperinflation produces an uneven distribution of blood flow, the "slow compartment" being permanently in zone II condition (i.e. alveolar pressure greater than pulmonary venous pressure), a setting impairing blood flow in the adjacent vascular area, and increasing global outflow impedance for the right ventricle (fig. 6). As previously stated, application of a low external PEEP suppresses the slow compartment [7] and thus reduces right ventricular outflow impedance [12]. This re-integration of the slow compartment in alveolar areas participating in tidal ventilation, and supplied with an adequate blood flow, produces a marked improvement in arterial oxygenation [7, 12].

\section{Conclusion}

At a time when a large cooperative study, with a major impact factor, advocates the beneficial effect of increasing respiratory rate during protective ventilation in acute respiratory distress syndrome patients, it appears important to put critical care intensivists on their guard against a systematic application of this debatable strategy [16]. First of all, a well-known notion should be remembered [17]: a high respiratory rate is totally inefficient in improving carbon dioxide in an adult patient [4], except when this strategy is accompanied by instrumental dead space reduction [18]. From a practical point of view, this implies that the heat and moisture exchanger should be replaced by a heated humidifier before increasing respiratory rate. Second, diffuse or localised airflow limitation is present in a majority of acute respiratory distress syndrome patients, and increasing respiratory rate usually causes dynamic hyperinflation, without any beneficial
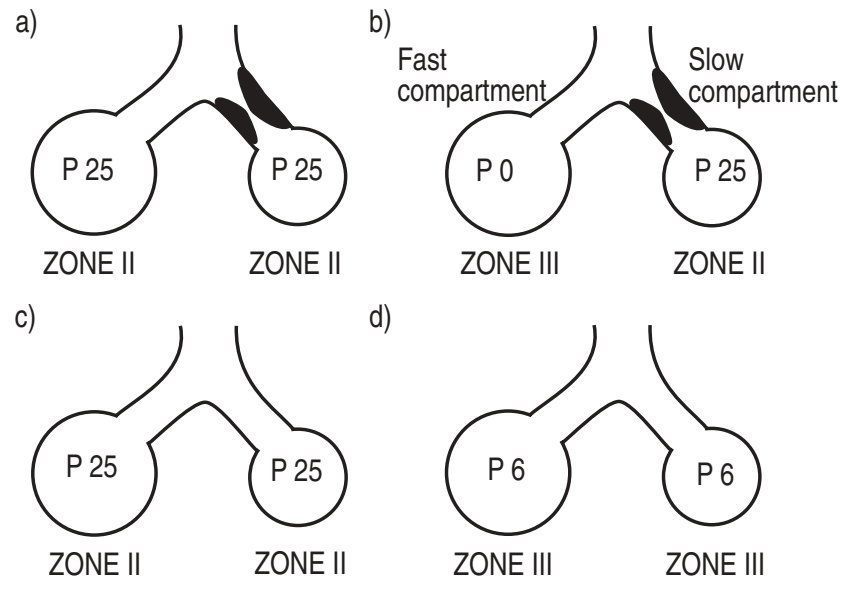

d)

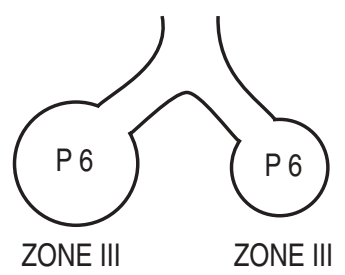

Fig. 6.-A schematic representation of the adverse haemodynamic effect of the slow compartment in acute respiratory distress syndrome. a) Tidal ventilation with zero end-expiratory pressure (ZEEP) produces a plateau pressure of $25 \mathrm{cmH}_{2} \mathrm{O}$, which creates a zone II condition. b) Airway pressure in the fast compartment returns to zero at end-expiration with ZEEP, restoring a zone III condition, whereas the "slow compartment", which cannot empty, is responsible for a permanent zone II condition in the corresponding vascular area. c) Tidal ventilation with PEEP also creates a zone II condition, d) but the low PEEP of $6 \mathrm{cmH}_{2} \mathrm{O}$ suppresses the "slow compartment", so that no zone II condition persists at expiration. 
effect on gas exchange, and with an adverse effect on pulmonary blood flow [4]. Thus, before deciding this strategy, intrinsic positive end-expiratory pressure should be carefully measured during a prolonged end-expiratory pause, and should be checked regularly if the strategy is implemented.

\section{References}

1. Pepe P, Marini J. Occult positive end-expiratory pressure in mechanically ventilated patients with airflow obstruction. Am Rev Respir Dis 1982; 126: 166-170.

2. Koutsoukou A, Armaganidis A, Stravrakaki-Kallergi C, et al. Expiratory flow limitation and intrinsic positive end-expiratory pressure at zero positive end-expiratory pressure in patients with adult respiratory distress syndrome. Am J Respir Crit Care Med 2000; 161: 1590-1596.

3. De Durante G, Del Turco M, Rustichini L, et al. ARDSNet lower tidal volume ventilatory strategy may generate intrinsic positive end-expiratory pressure in patients with acute respiratory distress syndrome. Am J Respir Crit Care Med 2002; 165: 1271-1274.

4. Vieillard-Baron A, Prin S, Augarde R, et al. Increasing respiratory rate to improve $\mathrm{CO}_{2}$ clearance during mechanical ventilation is not a panacea in acute respiratory failure Crit Care Med 2002; 30: 1407-1412.

5. Richard JC, Brochard L, Breton L, et al. Influence of respiratory rate on gas trapping during low volume ventilation of patients with acute lung injury. Intensive Care Med 2002; 28: 1078-1083.

6. Rossi A, Polese G, Brandi G, Conti G. Intrinsic positive end-expiratory pressure. Intensive Care Medicine 1995; 21: $522-536$

7. Vieillard-Baron A, Prin S, Schmitt JM, et al. Pressure/ volume curves in acute respiratory distress syndrome: clinical demonstration of the influence of expiratory flow limitation on the initial slope. Am J Respir Crit Care Med 2002; 165: 1107-1112.

8. Bilen Z, Cohen I. "Pseudo auto-PEEP"? A new cause for discrepancy between the end-expiratory occlusion plateau pressure and airway opening pressure. Chest 1993; 103: 1489-1494.
9. Rossi A, Gottfried S, Zocchi L, et al. Measurement of static compliance of the total respiratory system in patients with acute respiratory failure during mechanical ventilation. $\mathrm{Am}$ Rev Respir Dis 1983; 131: 672-678.

10. Suter P, Fairley B, Isenberg M. Optimum end-expiratory airway pressure in patients with acute pulmonary failure. N Engl J Med 1975; 292: 284-289.

11. Radford EP Jr. Mechanical properties of mammalian lungs. $I n$ : Fenn WO, Rahn $\mathrm{H}$, eds. Handbook of Physiology. Washington, DC, Am Physiol Society, 1964.

12. Schmitt JM, Vieillard-Baron A, Augarde R, Prin S, Page B, Jardin F. PEEP titration in ARDS patients: impact on right ventricular outflow impedance evaluated by pulmonary artery Doppler flow velocity. Critical Care Med 2001; 29: 1154-1158.

13. Mercat A, Titiriga M, Anguel N, Richard C, Teboul JL. Inverse ratio ventilation $(\mathrm{I} / \mathrm{E}=1 / 2)$ in acute respiratory distress syndrome: a six hours controlled study. Am J Respir Crit Care Med 1997; 155: 1637-1642.

14. Ranieri M, Giuliani R, Cinnella G, et al. Physiologic effects of positive end-expiratory pressure in patients with chronic obstructive pulmonary disease during acute ventilatory failure and controlled mechanical ventilation. Am Rev Respir Dis 1993; 147: 5-13.

15. Guérin C, LeMasson S, De Varx R, Milic-Emili J, Fournier G. Small airway closure and positive end-expiratory pressure in mechanically ventilated patients with chronic obstructive pulmonary disease. Am J Respir Crit Care Med 1997; 155: 1949-1956.

16. Ventilation with lower tidal volumes as compared with traditional tidal volumes for acute lung injury and the acute respiratory distress syndrome. The Acute Respiratory Distress Syndrome Network. $N$ Engl J Med 2000; 342: 1301-1308.

17. Harrison B, Murray M, Holets S. All that's gold does not glitter: effects of an increase in respiratory rate on pulmonary mechanics and $\mathrm{CO}_{2}$ kinetics in acute respiratory failure. Crit Care Med 2002; 30: 1648-1649.

18. Richecoeur J, Lu Q, Vieira S, et al. Expiratory washout versus optimization of mechanical ventilation during permissive hypercapnia in patients with severe acute respiratory distress syndrome. Am J Respir Crit Care Med 1998; 160: 77-85. 